\title{
ENTOMOFAUNA DE LA RESERVA CIENTÍFICA ÉBANO VERDE, CORDILLERA CENTRAL, REPÚBLICA DOMINICANA
}

\author{
Daniel E. Perez-Gelabert \\ Integrated Taxonomic Information System (ITIS) and Department of Entomology, United States \\ National Museum of Natural History (USNM), Smithsonian Institution, P.O. Box 37012, Washington, \\ DC 20013-7012, USA.perezd@si.edu
}

\begin{abstract}
RESUMEN
Se compila una lista preliminar de las especies de insectos conocidas para la Reserva Científica Ébano Verde (RCEV), Cordillera Central, República Dominicana, a partir de datos publicados en la literatura entomológica y el estudio de recolectas recientes. La lista incluye un total de 350 especies de insectos, clasificadas en 254 géneros, 70 familias y 12 órdenes. Cuatro especies de Syrphidae (Diptera) y una especie de Vespidae (Hymenoptera) constituyen nuevos registros para la República Dominicana y La Española. Estos datos, obviamente, abarcan solo una pequeña porción de la entomofauna total que habita el área. Los mismos deberán servir para impulsar otros estudios entomológicos en la zona y a su vez contribuir con el avance del conocimiento de los insectos en la Cordillera Central dominicana y de otras áreas protegidas de la isla.
\end{abstract}

Palabras clave: insectos, diversidad biológica, inventario, áreas protegidas, La Española, Antillas Mayores.

Title: Entomofauna of Reserva Científica Ébano Verde, La Vega Province, Dominican Republic.

\section{ABSTRACT}

A preliminary list of the insect species reported for the Reserva Científica Ébano Verde (RCEV), Cordillera Central, Dominican Republic, is compiled from the published entomological literature and the study of recent collections. The list includes a total of 350 species of insects classified in 254 genera, 70 families and 12 orders. Four species of Syrphidae (Diptera) and one species of Vespidae (Hymenoptera) are new records for the Dominican Republic and Hispaniola. Obviously these data represent only a small portion of the total entomofauna that inhabits the area. They should be useful to propitiate other entomological studies in the reserve and advance the knowledge of insects in the Dominican Cordillera Central and other protected areas of the island.

Keywords: insects, biological diversity, inventory, protected areas, Hispaniola, Greater Antilles.

\section{INTRODUCCIÓN}

Los insectos comprenden los grupos faunísticos más diversos en la mayoría de ecosistemas terrestres y estos pueden tener papeles importantes en los procesos ecosistémicos, siendo fundamentales para el funcionamiento y sostenibilidad de las comunidades naturales. Un mejor conocimiento de la entomofauna de una comunidad será cada vez más determinante para lograr su conservación. A nivel mundial se estima que se conocen alrededor de un millón de especies de insectos (Chapman, 2009), pero también que existe varias veces este número todavía por descubrir y bautizar con un nombre científico. La primera cuantificación de los insectos en La Española registró un total de 5,676 especies vivientes (Perez-Gelabert, 2008), total que 
actualmente sobrepasa las 6 mil especies. Un inventario comprensivo de la fauna entomológica de La Española representaría un paso muy significativo en el conocimiento de su biodiversidad.

La Reserva Científica Ébano Verde (RCEV) es una de las más de 100 áreas protegidas de la República Dominicana. Esta fue creada por el decreto presidencial No. 417-89 del 26 de octubre de 1989. La Ley General de Medio Ambiente y Recursos Naturales (64-00) y la Ley Sectorial de Areas Protegidas No. 202-04 ratifican la condición de área protegida de esta zona, con el mismo nombre, categoría y extensión. La Fundación para el Mejoramiento Humano (PROGRESSIO) es responsable del manejo y administración de la RCEV, bajo la modalidad de comanejo, mediante un acuerdo de fideicomiso con el Ministerio de Medio Ambiente y Recursos Naturales. Este modelo exitoso de gestión pública - privada compartida se considera un ejemplo para el manejo de otras áreas protegidas en el país.

Esta reserva constituye un reservorio de recursos hídricos de gran importancia para el Cibao Central, ya que sus elevaciones reciben un alto nivel de precipitaciones y albergan los nacimientos de varios ríos que suplen la necesidad de agua a varias ciudades y presas. Igualmente, esta reserva contiene importantes reductos del árbol endémico que da nombre a la misma, el ébano verde (Magnolia pallescens Urban \& Ekman, 1931). Antes de la explotación indiscriminada de su madera, esta especie estaba bien representada en el área. Actualmente, el ébano verde se encuentra en peligro de extinción y su recuperación es el objetivo de programas que incluyen su estudio, propagación y reintroducción en el área. El fácil acceso a la RCEV desde Santo Domingo y ciudades vecinas, además del respaldo a estudios biológicos por parte de la administración, incentivan los estudios de biodiversidad en esta área protegida. Hasta la fecha, los inventarios biológicos realizados en la RCEV incluyen la caracterización de su flora (García et al., 1994), lepidópteros (Domínguez, 1997), hongos basidiomycetes (Quírico Batista, 2004) y musgos (Mateo Jiménez, 2011). También se han publicado múltiples estudios botánicos donde se caracterizan las asociaciones vegetales encontradas en esta área (Castillo Torres et al., 1997) y estudios sobre las posibilidades de restauración de plantas nativas en los helechales de la reserva (Navarro et al., 2006).

El presente trabajo pretende unificar mediante una lista de especies el conocimiento ya existente sobre las especies que conforman la entomofauna de la RCEV. Un mejor entendimiento de esta fauna deberá promover investigaciones complementarias que permitan comprender de manera integrada la diversidad entomológica que habita esta región y promover un compromiso por parte de las nuevas generaciones de biólogos locales sobre la importancia de estos inventarios en las áreas protegidas de la isla.

\section{OBJETIVO}

- Producir un inventario de la diversidad conocida de los insectos que habitan la RCEV, Cordillera Central, República Dominicana.

\section{MATERIALES Y MÉTODOS}

Área de Estudio. La Reserva Científica Ébano Verde se ubica en la porción oriental de la Cordillera Central, entre las coordenadas geográficas $19^{\circ} 06^{\prime}$ latitud Norte y $70^{\circ} 33^{\prime}$ longitud Oeste (Fig. 1). La misma tiene una extensión de $29.81 \mathrm{~km}^{2}$ (2,981 ha.). La geología del área, como todo el centro de la isla corresponde a rocas volcánicas y su relieve se ubica entre alturas de 800 - 1,565 msnm, siendo su mayor altura la Loma La Golondrina. La reserva recibe un promedio anual de $3,100 \mathrm{~mm}$ de lluvia y mantiene una temperatura promedio anual de $17{ }^{\circ} \mathrm{C}$. La zona de vida presente en esta área es de tipo Bosque Muy Húmedo Montano Bajo en un 
93\% de su área núcleo e incluye bosques de manaclas (Prestoea acuminata (Wild.) H. E. Moore var. montana (Graham) A. J. Hend. \& Galeano), bosque nublado y pinos (Pinus occidentalis Swartz, 1788) y plantaciones de pino hondureño (Pinus caribaea Morelet, 1851), con gran abundancia de helechos, plantas epífitas y briofitas. Su flora incluye 688 especies de plantas vasculares, mientras que su fauna está compuesta por importantes especies nativas y endémicas de vertebrados, incluyendo poblaciones del mamífero insectívoro Solenodon paradoxus Brandt, 1833, así como de 90 especies de aves, ocho especies de anfibios y varias especies de reptiles (Castillo Torres et al., 1997).

Análisis de la literatura e Inventario de especies. Para detectar las especies de insectos citadas previamente de esta reserva, se realizó un examen intensivo de la literatura entomológica mundial. Se anotaron las localidades donde se recolectaron las especies, el autor de su nombre científico y el número de página en la referencia en que las especies se citan para la RCEV. Se incluyen especies quizás no exactamente colectadas dentro de los terrenos de la reserva pero citados en la literatura en asociación a la Loma Casabito. Igualmente, se identificaron especímenes recolectados principalmente por el autor en múltiples visitas a la RCEV durante los últimos 20 años y mantenidos en las colecciones del United States National Museum of Natural History, Smithsonian Institution, Washington, DC (USNM), el Museo Nacional de Historia Natural "Prof. Eugenio de Jesús Marcano" (MNHNSD), en Santo Domingo y el Instituto de Investigaciones Botánicas y Zoológicas (IIBZ) de la Universidad Autónoma de Santo Domingo. Muchos de estos especímenes fueron recolectados por el autor y colaboradores entre 20022004 durante un proyecto que perseguía el inventario de los insectos ortopteroides en República Dominicana. En general, los insectos fueron capturados usando una red entomológica o en una sábana iluminada con luz UV.

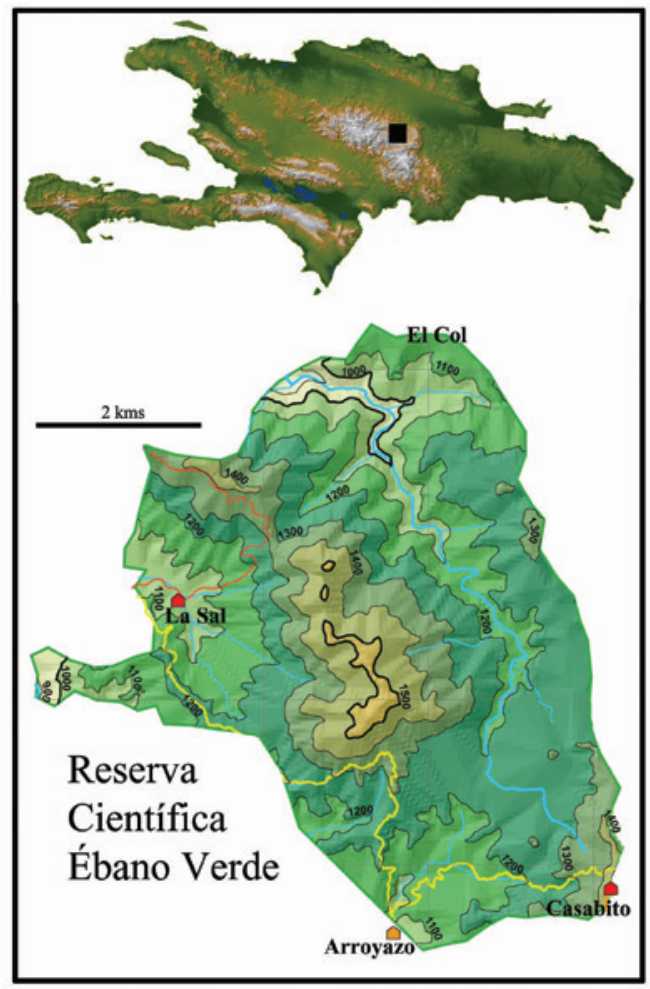




\section{RESULTADOS Y DISCUSIÓN}

Se compilan registros de un total de 350 especies de insectos repartidas en 254 géneros, 70 familias y 12 órdenes de los 29 conocidos para La Española (Tablas I y II; Figs. 2 y 3 ). Esto representa un poco más del 5\% de las más de 6,000 especies de insectos ya registradas para la isla. La mayor cantidad de especies pertenecen al orden Lepidoptera (143), seguidos de Coleoptera (54) y Orthoptera (37). Dentro de los Diptera, cuatro especies de Syrphidae (Allograpta neotropica Curran, Toxomerus lacrymosus (Bigot), Toxomerus pictus (Macquart) y Toxomerus violaceus Curran) son citadas aquí como nuevos registros para el país y la isla. También se cita por primera vez la avispa social Mischocyttarus mexicanus cubicola Richards, 1978. Estas especies son marcadas con un asterisco en la Tabla II. Solo unas pocas de estas especies son hasta ahora conocidas únicamente del territorio de la RCEV, aunque probablemente sus poblaciones se extiendan a territorios adyacentes, especialmente a los mismos niveles altitudinales de la Cordillera Central.

Los primeros muestreos de insectos en Ébano Verde ocurrieron antes de que el área fuese declarada protegida. Los primeros registros de insectos son los de Campbell (1978) sobre escarabajos tenebriónidos, los de Baroni Urbani (1978) sobre hormigas y Howden (1978) sobre escarabajos (Scarabaeidae). Los insectos fueron recolectados en el área de la Loma de Casabito, que ya era accesible por su cercanía a la carretera que conduce a Constanza y por la construcción de antenas de radio/televisión en su cima. Múltiples colectores han visitado el área de las antenas en la Loma Casabito y también el área de El Arroyazo donde se encuentran las instalaciones administrativas de la reserva.

El trabajo entomofaunístico más comprensivo publicado hasta ahora para la RCEV es el de Domínguez (1997). Este incluye una lista de especies de varias familias de lepidópteros. Hasta ahora esta era también la única publicación específica y exclusivamente dedicada a insectos de la RCEV. Pero este listado de lepidópteros presenta pocos datos sobre las especies o las localidades donde se recolectaron dentro de la reserva. En la Tabla II estas especies son listadas como recolectadas en la "Reserva Ébano Verde". Con la finalidad de hacer esta información lo más útil posible, se utilizan los nombres actualizados de las especies. Los nombres inválidos citados por Domínguez (1997), son incluidos bajo el nombre actual de la especie. Como era de esperar por ser las especies de lepidópteros con mayor probabilidad de ser identificadas, la mayoría son especies de amplia distribución en la isla y el Caribe. Se destacan los lepidópteros nocturnos de la familia Sphingidae con 27 (50.9\%) de las 53 especies registradas para La Española.

Los lepidópteros son el contingente más grande en la lista, con 143 especies (40.9\%), distribuidas en 10 familias. En este grupo se encuentran muchas especies que por tener larvas fitófagas, afectan la producción agrícola. Algunas son especies de poca importancia económica, mientras otras son plagas de mayor importancia y contra las cuales los productores agrícolas deben siempre adoptar medidas de control. Entre los coleópteros los datos están limitados a 54 especies (14.3\%) repartidas en 15 familias. Entre los más llamativos hay nueve especies de luciérnagas (Lampyridae) que con sus destellos de bioluminescencia se hacen notar durante las noches. Estas se asocian principalmente con la vegetación cercana a las cañadas. También se conocen nueve especies de la familia Cerambycidae y 12 especies de Scarabaeidae, nueve de ellas en el género Phyllophaga Harris. Los dípteros incluyen cinco familias con citas de especies. Los mayes (Simuliidae) al igual que los mosquitos (Culicidae) están entre los insectos más conspicuos en la reserva pues pueden ser molestos picadores diurnos de los visitantes humanos. Se identificaron dos especies de mosquitos con hábitos similares (Howardina albonotata (Coquillett) y Wyeomyia (Wyeomyia) mitchellii (Theobald)) ambas colectadas en el área de El Arroyazo. Los sírfidos o moscas de las flores, pueden ser importantes polinizadores. La lista incluye 16 especies de Syrphidae, incluyendo cuatro nuevos registros. De los hemípteros, se 
encuentran especies fitófagas como los hiedevivos (Pentatomidae) y también entomófagas como las chinches asesinas (Reduviidae). La lista incluye nueve familias, entre las cuales sobresalen las chicharritas (Cicadellidae) con seis especies y los Lygaeidae con 12 especies. Los himenópteros han sido poco estudiados $\mathrm{y}$ aparecen representados por solo seis familias con unas pocas especies cada una. Una avispa endémica de la isla y hasta ahora conocida de un solo especimen recolectado en la RCEV es Sirex hispaniola Goulet et al., 2012 (Siricidae), que son avispas no sociales y xilófagas (comen madera). Este es el único miembro conocido de esta familia de avispas en La Española. Entre los ortópteros se incluyen especies de cuatro familias. Los más destacados son los grillos, de los cuales se han citado 20 especies pertenecientes a varios géneros. Entre los grupos cercanos a los ortópteros, la lista incluye nueve especies de cucarachas (Blattodea). Las mantis (Mantodea) y mariapalitos (Phasmatodea) solo incluyen unas pocas especies hasta ahora identificadas. Entre los insectos acuáticos los únicos órdenes incluidos en la lista son los Trichoptera, representados por 13 especies de ocho familias y los Odonata (libélulas y damiselas), representados por 17 especies de siete familias. Ambos grupos tienen larvas depredadoras que viven entre las piedras y las corrientes de agua y son componentes importantes de la red trófica en los ecosistemas acuáticos.

Aunque en años recientes varias áreas protegidas empiezan a ser objeto de inventarios faunísticos que incluyen artrópodos, esto todavía se hace principalmente para el grupo más conocido y fácil de identificar que son las mariposas, e. g., Hilario Pérez (2013), en el Parque Aniana Vargas y Rodríguez et al. (2014) en el Parque Nacional Sierra Martín García. Esta carencia en parte se relaciona con la baja disponibilidad de expertos que puedan identificar otros grupos de insectos. La primera área protegida en la República Dominicana para la cual se compiló un listado preliminar de sus insectos fue el Parque Nacional Loma Nalga de Maco (Perez-Gelabert et al., 2011) localizado en el extremo noroeste de la Cordillera Central, de donde se inventariaron 133 especies pertenecientes a nueve órdenes. Sorprendentemente, solo 22 de las especies listadas aquí para la RCEV fueron también citadas para el Parque Nacional Loma Nalga de Maco. Esto parece deberse a que gran parte de los registros de ambas áreas se hicieron como parte de trabajos taxonómicos independientes enfocados al descubrimiento de especies nuevas para la ciencia. Por otro lado, mientras que los lepidópteros reportados de Ébano Verde incluyen varias familias de lepidópteros diurnos y nocturnos, los reportados de Nalga de Maco fueron casi exclusivamente mariposas.

Toda la fauna de invertebrados acuáticos en la República Dominicana ha sido poco atendida por los investigadores nativos (y aun los extranjeros) tanto desde el punto de vista taxonómico como en evaluaciones ecológicas. De la misma manera, los insectos acuáticos en la RCEV han sido poco documentados y muchos insectos comunes quedan por ser registrados. Múltiples grupos de insectos acuáticos como Ephemeroptera, hemípteros y coleópteros acuáticos todavía no han sido estudiados en la reserva. Un trabajo donde se investigó la ecología de macroinvertebrados de agua dulce en la República Dominicana es de Soldner et al. (2004), quienes realizan una evaluación de diferentes cuerpos de agua en la cuenca del río Yaque del Norte. Estos autores hallaron correlación entre la estructura de los ensamblajes de macroinvertebrados y la calidad del agua, aunque no fue claro si esto se debía a variables ambientales como la altura o era debido a la contaminación. En un reciente análisis comparativo de la fauna de insectos acuáticos en las Antillas Mayores (Gutiérrez-Fonseca et al., 2013) reportaron la mayor riqueza para La Española con 63 familias, seguida de Puerto Rico con 61 y Cuba con 60 familias. Dado el estado de conocimiento tan preliminar y que en La Española se espera encontrar algunas familias más aun no citadas, esto es algo sorprendente y a la vez un indicador de la relativamente alta diversidad de artrópodos en esta isla. 


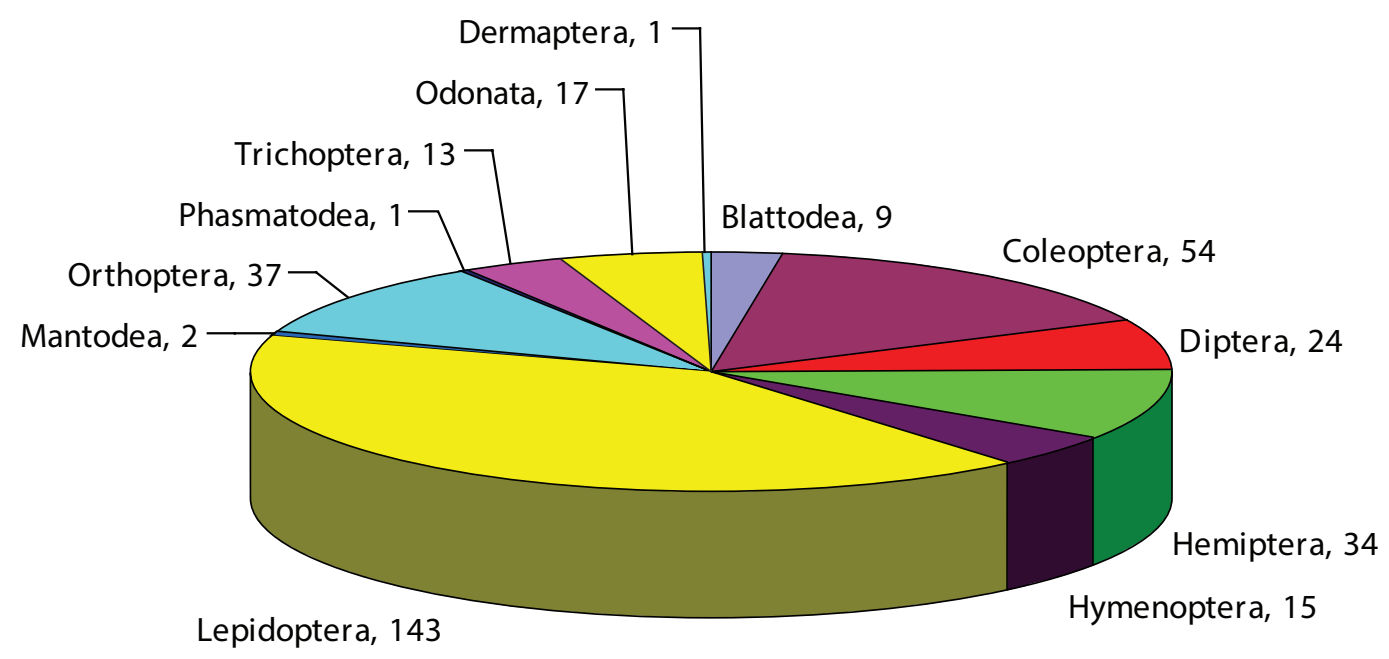

Figura 2. Diversidad de especies por órdenes de insectos inventariadas para la RCEV. El total es de 350 especies.

Tabla I. Números de especies por órdenes de insectos inventariados para la RCEV.

\begin{tabular}{|c|c|c|c|}
\hline Órdenes & Familias & Géneros & Especies \\
\hline Blattodea & 3 & 8 & 9 \\
\hline Coleoptera & 15 & 38 & 54 \\
\hline Dermaptera & 1 & 1 & 24 \\
\hline Diptera & 5 & 14 & 34 \\
\hline Hemiptera & 9 & 28 & 15 \\
\hline Hymenoptera & 6 & 13 & 143 \\
\hline Lepidoptera & 10 & 99 & 17 \\
\hline Mantodea & 1 & 2 & 37 \\
\hline Odonata & 7 & 13 & 13 \\
\hline Orthoptera & 4 & 29 & 350 \\
\hline Phasmatodea & 1 & 254 & 1 \\
\hline Trichoptera & 8 & & 8 \\
\hline Total & 70 & 89 & 13 \\
\hline
\end{tabular}



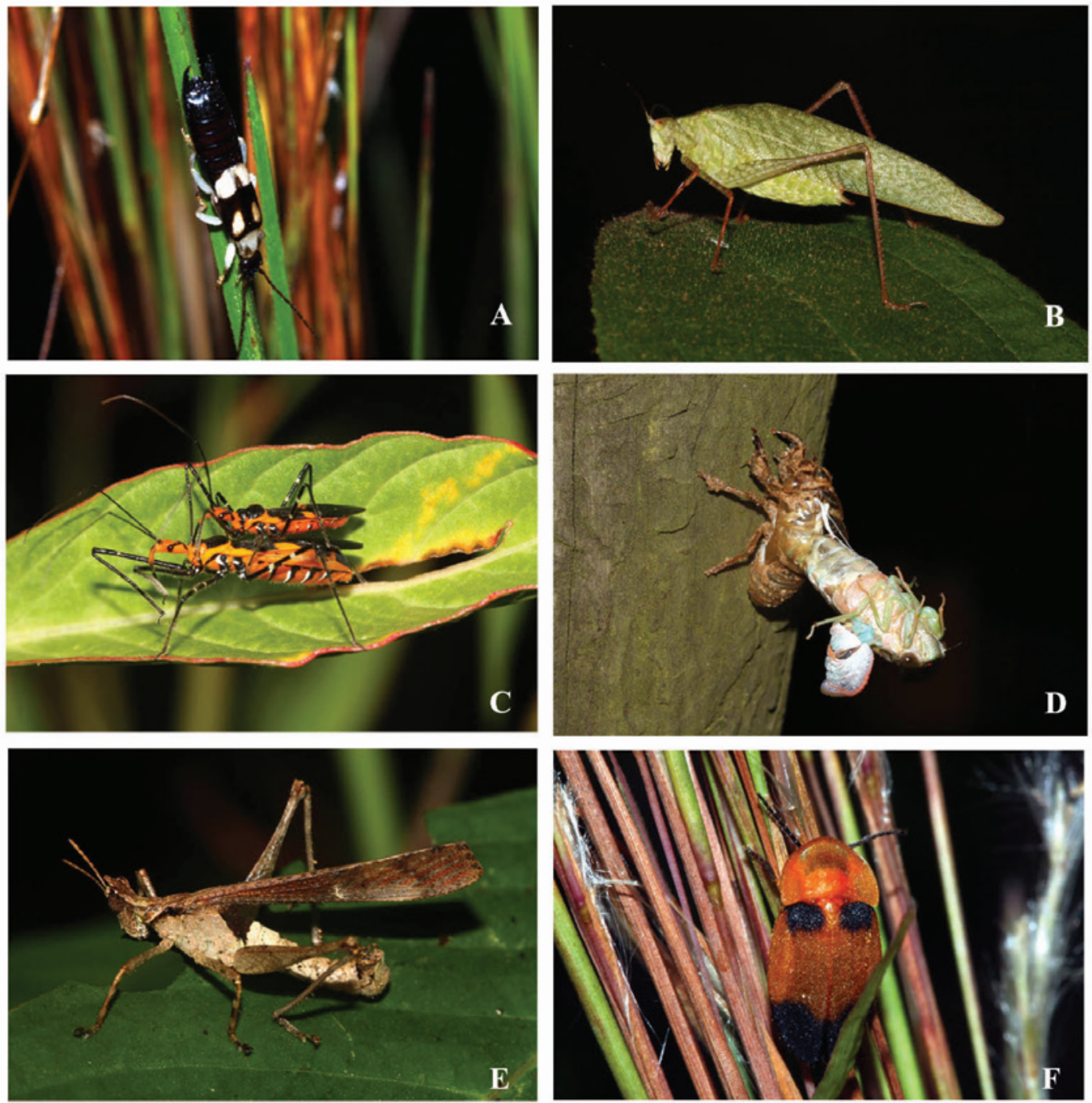

Figura 3, A-F. Fotos de algunos insectos encontrados en la RCEV. A, Doru albipes (Dermaptera: Forficulidae). B, Turpilia sp. (Orthoptera: Tettigoniidae). C, Zelus longipes (Hemiptera: Reduviidae). D, muda de chicharra (Hemiptera: Cicadidae). E, Espagnola darlingtoni (Orthoptera: Episactidae). F, Callopisma dominicana (Coleoptera: Lampyridae). 
Tabla II. Lista de especies de insectos inventariados para la Reserva Científica Ébano Verde.

\begin{tabular}{|c|c|c|c|c|c|c|c|}
\hline Especies & \begin{tabular}{|l|} 
El \\
Arroyazo
\end{tabular} & $\begin{array}{l}\text { Loma } \\
\text { Casabito }\end{array}$ & La Sal & El Col & $\begin{array}{l}\text { Loma La } \\
\text { Golondrina }\end{array}$ & RCEV & Referencia \\
\hline \multicolumn{8}{|l|}{ BLATTODEA } \\
\hline \multicolumn{8}{|l|}{ BLABERIDAE } \\
\hline Colapteroblatta sp. & - & & & & & & Det. Perez-Gelabert (USNM) \\
\hline Epilampra sp. & $\bullet$ & & & & & & Det. Perez-Gelabert (USNM) \\
\hline Neoblattella proserpina Rehn \& Hebard & & & $\bullet$ & & & & $\begin{array}{l}\text { Gutiérrez \& Perez-Gelabert, } \\
\text { 2000: } 437\end{array}$ \\
\hline Symploce bicolor (Palisot de Beauvois) & & $\bullet$ & & & & & $\begin{array}{l}\text { Gutiérrez \& Perez-Gelabert, } \\
\text { 2000: } 434\end{array}$ \\
\hline \multicolumn{8}{|l|}{ BLATELLIDAE } \\
\hline Caribblatta sp. & $\bullet$ & & & & & & Det. Perez-Gelabert (USNM) \\
\hline Euthlastoblatta sp. & & & & $\bullet$ & & & Det. Perez-Gelabert (USNM) \\
\hline Plectoptera sp. & & & & $\bullet$ & & & Det. Perez-Gelabert (USNM) \\
\hline \multicolumn{8}{|l|}{ BLATTIDAE } \\
\hline Eurycotis hebardi Gutiérrez & & $\bullet$ & & & & & Gutiérrez, 2013: 26 \\
\hline Eurycotis rehni Gutiérrez & - & - & - & & & & Gutiérrez, 2013: 29 \\
\hline \multicolumn{8}{|l|}{ COLEOPTERA } \\
\hline \multicolumn{8}{|l|}{ CANTHARIDAE } \\
\hline Tytthonyx (Tytthonyx) mutabilis Wittmer & & $\bullet$ & & & & & Wittmer, 1992: 246 \\
\hline \multicolumn{8}{|l|}{ CERAMBYCIDAE } \\
\hline $\begin{array}{l}\text { Byrandra (Yvesandra) laevis Santos-Silva } \\
\& \text { Shute }\end{array}$ & & & & $\bullet$ & & & Det. Perez-Gelabert (IIBZ) \\
\hline Calocosmus nigritarsis Fisher & & & & - & & & Det. Perez-Gelabert (IIBZ) \\
\hline Caribbomerus elongatus (Fisher) & & - & & & & & Lingafelter, 2011: 29 \\
\hline Derancistrus anthracinus (Gahan) & & $\bullet$ & & & & & Det. Perez-Gelabert (IIBZ) \\
\hline Leptostylopsis viridicomus (Fisher) & & $\bullet$ & & & & & Lingafelter \& Micheli, 2009: 27 \\
\hline Methia dolichoptera Lingafelter & & $\bullet$ & & & & & Lingafelter, 2010: 266 \\
\hline Novantinoe darlingtoni (Fisher) & $\bullet$ & & $\bullet$ & & & & Det. Perez-Gelabert (IIBZ) \\
\hline Prionus aureopilosus Fragoso \& Monné & & $\bullet$ & & & & & Det. Perez-Gelabert (IIBZ) \\
\hline Sarifer seabrai Fragoso \& Monné & & & & & & $\bullet$ & Fernández, 2010: 146 \\
\hline \multicolumn{8}{|l|}{ CHRYSOMELIDAE } \\
\hline Ceratoma ruficornis (Olivier) & $\bullet$ & & & & & & Det. Perez-Gelabert (USNM) \\
\hline Distigmoptera antennata Medvedev & & - & & & & & Medvedev, 2004: 122 \\
\hline Elytrogona quatuordecimmaculata Latreille & & - & & & & & Chaboo, 2000: 389 \\
\hline Homoschema sp. & & & & $\bullet$ & & & Det. Perez-Gelabert (USNM) \\
\hline Leptophysa hispaniolae (Blake) & & $\bullet$ & & & & & Medvedev, 2004: 124 \\
\hline Leptophysa ornata Medvedev & & $\bullet$ & & & & & Medvedev, 2004: 124 \\
\hline \multicolumn{8}{|l|}{ CLERIDAE } \\
\hline Decorosa aladecoris Opitz & & - & & & & & Opitz, 2008: 6 \\
\hline \multicolumn{8}{|l|}{ COCCINELLIDAE } \\
\hline Cycloneda sanguinea (Linnaeus) & & & & $\bullet$ & & & Det. Perez-Gelabert (USNM) \\
\hline \multicolumn{8}{|l|}{ CURCULIONIDAE } \\
\hline Euscelus sp. & & & & $\bullet$ & & & Det. Perez-Gelabert (USNM) \\
\hline Metamasius hemipterus (Olivier) & & & & $\bullet$ & & & Det. Perez-Gelabert (USNM) \\
\hline Peridinetus concentricus (Olivier) & $\bullet$ & & & & & & Prena, 2009: 56 \\
\hline \multicolumn{8}{|l|}{ EROTYLIDAE } \\
\hline Notaepitus fulvitarsis (Lacordaire) & & - & & & & & Skelley, 2009: 94 \\
\hline \multicolumn{8}{|l|}{ LAMPYRIDAE } \\
\hline $\begin{array}{l}\text { Callopisma dominicana Kazantsev \& } \\
\text { Perez-G. }\end{array}$ & $\bullet$ & $\bullet$ & & & & & $\begin{array}{l}\text { Kazantsev \& Perez-Gelabert, } \\
\text { 2009: } 376\end{array}$ \\
\hline Erythrolychnia bipartita (Olivier) & & & $\bullet$ & & & & $\begin{array}{l}\text { Kazantsev \& Perez-Gelabert, } \\
\text { 2009: } 380\end{array}$ \\
\hline $\begin{array}{l}\text { Erythrolychnia roseimargo Kazantsev \& } \\
\text { Perez-G. }\end{array}$ & & & & & & & $\begin{array}{l}\text { Kazantsev \& Perez-Gelabert, } \\
\text { 2009: } 385\end{array}$ \\
\hline
\end{tabular}


Tabla II (continuación).

\begin{tabular}{|c|c|c|c|c|c|c|c|}
\hline Especies & $\begin{array}{l}\text { El } \\
\text { Arroyazo }\end{array}$ & $\begin{array}{l}\text { Loma } \\
\text { Casabito }\end{array}$ & La Sal & El Col & $\begin{array}{l}\text { Loma La } \\
\text { Golondrina }\end{array}$ & RCEV & Referencia \\
\hline Heterophotinus constanzae Kazantsev & $\bullet$ & & - & & & & $\begin{array}{l}\text { Kazantsev \& Perez-Gelabert, } \\
\text { 2009: } 394\end{array}$ \\
\hline Heterophotinus glaucus (Olivier) & $\bullet$ & & $\bullet$ & & & & $\begin{array}{l}\text { Kazantsev \& Perez-Gelabert, } \\
\text { 2009: } 394\end{array}$ \\
\hline $\begin{array}{l}\text { Heterophotinus quadrinotatus (Motschul- } \\
\text { sky) }\end{array}$ & & & $\bullet$ & & $\bullet$ & & $\begin{array}{l}\text { Kazantsev \& Perez-Gelabert, } \\
\text { 2009: } 396\end{array}$ \\
\hline Heterophotinus viridicolor Kazantsev & - & & & & - & & $\begin{array}{l}\text { Kazantsev \& Perez-Gelabert, } \\
\text { 2009: } 396\end{array}$ \\
\hline Heterophotinus vittatus (Olivier) & $\bullet$ & & & & & & $\begin{array}{l}\text { Kazantsev \& Perez-Gelabert, } \\
\text { 2009: } 397\end{array}$ \\
\hline Presbyolampis vegaensis Kazantsev & $\bullet$ & & $\bullet$ & & & & $\begin{array}{l}\text { Kazantsev \& Perez-Gelabert, } \\
\text { 2009: } 399\end{array}$ \\
\hline \multicolumn{8}{|l|}{ LYCIDAE } \\
\hline Thonalmus bicolor (Linnaeus) & & & & $\bullet$ & & & Det. Perez-Gelabert (USNM) \\
\hline \multicolumn{8}{|l|}{ MELOIDAE } \\
\hline Tetraonyx quadrimaculata (Fabricius) & & & & & & - & $\begin{array}{l}\text { Woodruff \& Baranowski (No } \\
\text { publicado) }\end{array}$ \\
\hline \multicolumn{8}{|l|}{ PASSALIDAE } \\
\hline Passalus dominicanus van Doesburg & $\bullet$ & & & & & & Det. Perez-Gelabert (USNM) \\
\hline \multicolumn{8}{|l|}{ RHYSODIDAE } \\
\hline $\begin{array}{l}\text { Clinidium (Clinidium) trionyx } \mathrm{R} . \text { Bell \& } \\
\text { J. Bell }\end{array}$ & & $\bullet$ & & & & & R. Bell \& J. Bell, 1985: 124 \\
\hline \multicolumn{8}{|l|}{ SCARABAEIDAE } \\
\hline Ataenius heinekeni (Wollaston) & & $\bullet$ & & & & & Stebnicka, 2004: 216 \\
\hline Cyclocephala sp. & & & & $\bullet$ & & & Det. Perez-Gelabert (USNM) \\
\hline $\begin{array}{l}\text { Hispanioryctes wittmeri Howden \& En- } \\
\text { drödi }\end{array}$ & & $\bullet$ & & & & & Howden, 1978: 389 \\
\hline Phyllophaga aliada Sanderson & $\bullet$ & & $\bullet$ & $\bullet$ & & & Det. S. Medrano (IIBZ, USNM) \\
\hline Phyllophaga canoa Sanderson & & $\bullet$ & & & & & Woodruff \& Sanderson, 2004: 54 \\
\hline Phyllophaga eladio Woodruff & & & & $\bullet$ & & & Det. S. Medrano (IIBZ) \\
\hline $\begin{array}{l}\text { Phyllophaga jimenezi Woodruff \& Sand- } \\
\text { erson }\end{array}$ & & $\bullet$ & $\bullet$ & & & & Det. S. Medrano (IIBZ) \\
\hline Phyllophaga kenscoffi (Wolcott) & & & $\bullet$ & & & & Det. S. Medrano (IIBZ) \\
\hline Phyllophaga mella Sanderson & & $\bullet$ & & & & & Woodruff \& Sanderson, 2004: 103 \\
\hline Phyllophaga n.sp. & $\bullet$ & & & $\bullet$ & & & Det. S. Medrano (IIBZ) \\
\hline Phyllophaga pseudocalcaris (Saylor) & $\bullet$ & & & & & & Det. S. Medrano (IIBZ) \\
\hline \multicolumn{8}{|l|}{ STAPHYLINIDAE } \\
\hline Euconnus abnormeantennatus Franz & & $\bullet$ & & & & & Franz, 1984: 18 \\
\hline Euconnus cazabitae Franz & & $\bullet$ & & & & & Franz, 1984: 20 \\
\hline Euconnus dominicanus Franz & & $\bullet$ & & & & & Franz, 1984: 20 \\
\hline Euconnus nigerrimus Franz & & $\bullet$ & & & & & Franz, 1984: 21 \\
\hline Microscydmus cazabitanus Franz & & $\bullet$ & & & & & Franz, 1984: 30 \\
\hline \multicolumn{8}{|l|}{ TENEBRIONIDAE } \\
\hline Lobopoda (Lobopoda) haitensis Campbell & & $\bullet$ & & & & & Campbell, 1978: 203 \\
\hline Loxostethus gowdeyi (Pic) & & $\bullet$ & & & & & Triplehorn \& Merk1, 1997: 739 \\
\hline Nesocyrtosoma otus Hopp \& Ivie & & & $\bullet$ & & & & Hopp \& Ivie, 2009: 45 \\
\hline \multicolumn{8}{|l|}{ DERMAPTERA } \\
\hline \multicolumn{8}{|l|}{ FORFICULIDAE } \\
\hline Doru albipes (Fabricius) & $\bullet$ & & & & & & Det. Perez-Gelabert (USNM) \\
\hline \multicolumn{8}{|l|}{ DIPTERA } \\
\hline \multicolumn{8}{|l|}{ ASILIDAE } \\
\hline $\begin{array}{l}\text { Beameromyia incisuralis Scarbrough \& } \\
\text { Page }\end{array}$ & & & & & $\bullet$ & & Scarbrough et al., 2005: 42 \\
\hline Ommatius bastardoanus Scarbrough & $\bullet$ & & $\bullet$ & & & & $\begin{array}{l}\text { Scarbrough \& Perez-Gelabert, } \\
\text { 2003: } 43\end{array}$ \\
\hline
\end{tabular}


Tabla II (continuación).

\begin{tabular}{|c|c|c|c|c|c|c|c|}
\hline Especies & \begin{tabular}{|l|} 
El \\
Arroyazo
\end{tabular} & $\begin{array}{l}\text { Loma } \\
\text { Casabito }\end{array}$ & La Sal & El Col & \begin{tabular}{|l|} 
Loma La \\
Golondrina
\end{tabular} & RCEV & Referencia \\
\hline Ommatius elusivus Scarbrough & $\bullet$ & & $\bullet$ & & & & $\begin{array}{l}\text { Scarbrough \& Perez-Gelabert, } \\
\text { 2003: } 46\end{array}$ \\
\hline Ommatius russelli Scarbrough & $\bullet$ & & $\bullet$ & & & & $\begin{array}{l}\text { Scarbrough \& Perez-Gelabert, } \\
\text { 2003: } 55\end{array}$ \\
\hline \multicolumn{8}{|l|}{ CULICIDAE } \\
\hline Howardina albonotata (Coquillett) & $\bullet$ & & & & & & Det. G. Guzmán (IIBZ) \\
\hline $\begin{array}{l}\text { Wyeomyia (Wyeomyia) mitchellii } \\
\text { (Theobald) }\end{array}$ & $\bullet$ & & & & & & Det. G. Guzmán (IIBZ) \\
\hline \multicolumn{8}{|l|}{ SIMULIIDAE } \\
\hline Simulium sp. & $\bullet$ & & & & & & Det. Perez-Gelabert (USNM) \\
\hline \multicolumn{8}{|l|}{ SYRPHIDAE } \\
\hline Allograpta (Antillus) ascita Vockeroth & & & & & $\bullet$ & & Det. C. Thompson (USNM) \\
\hline Allograpta neotropica Curran* & & & & & $\bullet$ & & Det. C. Thompson (USNM) \\
\hline Leucopodella gracilis (Williston) & $\bullet$ & & $\bullet$ & & $\bullet$ & & Det. C. Thompson (USNM) \\
\hline Meromacrus pinguis (Fabricius) & $\bullet$ & & & $\bullet$ & $\bullet$ & & Det. C. Thompson (USNM) \\
\hline Ocyptamus oriel (Hull) & & & & & $\bullet$ & & Det. C. Thompson (USNM) \\
\hline Ornidia obesa (Fabricius) & & & & $\bullet$ & & & Det. Perez-Gelabert (USNM) \\
\hline Palpada vinetorum (Fabricius) & & $\bullet$ & & & & & Det. C. Thompson (USNM) \\
\hline Rhinoprosopa aenea (Hull) & $\bullet$ & & $\bullet$ & & $\bullet$ & & Mengual, 2014: 5 \\
\hline Toxomerus arcifer (Loew) & & & $\bullet$ & & $\bullet$ & & Det. C. Thompson (USNM) \\
\hline Toxomerus dispar (Fabricius) & $\bullet$ & & $\bullet$ & & $\bullet$ & & Det. C. Thompson (USNM) \\
\hline Toxomerus floralis (Fabricius) & $\bullet$ & & $\bullet$ & & $\bullet$ & & Det. C. Thompson (USNM) \\
\hline Toxomerus lacrymosus (Bigot)* & & & $\bullet$ & & $\bullet$ & & Det. C. Thompson (USNM) \\
\hline Toxomerus pictus (Macquart)* & & & $\bullet$ & & $\bullet$ & & Det. C. Thompson (USNM) \\
\hline Toxomerus politus (Say) & & & & & $\bullet$ & & Det. C. Thompson (USNM) \\
\hline Toxomerus pulchellus (Macquart) & $\bullet$ & & & & & & Det. C. Thompson (USNM) \\
\hline Toxomerus violaceus Curran* & & & $\bullet$ & & $\bullet$ & & Det. C. Thompson (USNM) \\
\hline \multicolumn{8}{|l|}{ TACHINIDAE } \\
\hline Trichopoda sp. & & & & $\bullet$ & & & Det. Perez-Gelabert (USNM) \\
\hline \multicolumn{8}{|l|}{ HEMIPTERA } \\
\hline \multicolumn{8}{|l|}{ CICADELLIDAE } \\
\hline Agalliopsis longistyla Freytag & & $\bullet$ & & & & & Freytag, 2009: 271 \\
\hline Caribovia rubida Freytag & & $\bullet$ & & & & & Freytag, 2005b: 361 \\
\hline Curtara (Curtara) cornuta Freytag & & $\bullet$ & & & & & Freytag, 2005a: 327 \\
\hline Neohadria grisea Freytag & & $\bullet$ & & & & & Freytag, 2007: 395 \\
\hline Neokrisna decliva Freytag & & $\bullet$ & & & & & Freytag, 2012: 4 \\
\hline Neokrisna longula Freytag & & $\bullet$ & & & & & Freytag, 2012: 5 \\
\hline \multicolumn{8}{|l|}{ CICADIDAE } \\
\hline Chinaria viviana Ramos & $\bullet$ & & & & & & Fernández et al. 2007: 246 \\
\hline Odopoea cariboea Uhler & $\bullet$ & & & $\bullet$ & & & Det. Perez-Gelabert (USNM) \\
\hline Uhleroides hispaniolae Davis & & & & & & $\bullet$ & Fernández, 2007: 171 \\
\hline \multicolumn{8}{|l|}{ COREIDAE } \\
\hline Acanthocerus crucifer Palisot de Beauvois & $\bullet$ & & & $\bullet$ & & & Det. Perez-Gelabert (USNM) \\
\hline \multicolumn{8}{|l|}{ LYGAEIDAE } \\
\hline Xyonysius californicus (Stål) & & $\bullet$ & & & & & Baranowski \& Slater, 2005: 44 \\
\hline Kleidocerys hispaniola Baranowski & & & & & & & Baranowski \& Slater, 2005: 51 \\
\hline Kleidocerys viridescens (Fabricius) & & & & & & $\bullet$ & Baranowski \& Slater, 2005: 51 \\
\hline Ischnodemus variegatus (Signoret) & & & & & & $\bullet$ & Baranowski \& Slater, 2005: 63 \\
\hline Ninyas deficiens (Lethierry) & & & & & & $\bullet$ & Baranowski \& Slater, 2005: 68 \\
\hline Neopamphantus hispaniolus Baranowski & & & & & & $\bullet$ & Baranowski \& Slater, 2005: 75 \\
\hline Oncopeltus fasciatus (Dallas) & & & & $\bullet$ & & & Det. Perez-Gelabert (USNM) \\
\hline Ozophora auroseta Baranowski & & & & & & $\bullet$ & Baranowski \& Slater, 2005: 192 \\
\hline
\end{tabular}


Tabla II (continuación).

\begin{tabular}{|c|c|c|c|c|c|c|c|}
\hline Especies & $\begin{array}{l}\text { El } \\
\text { Arroyazo }\end{array}$ & $\begin{array}{l}\text { Loma } \\
\text { Casabito }\end{array}$ & La Sal & $\mathrm{El} \mathrm{Col}$ & $\begin{array}{l}\text { Loma La } \\
\text { Golondrina }\end{array}$ & RCEV & Referencia \\
\hline Ozophora cacumena Slater & & $\bullet$ & & & & & Baranowski \& Slater, 2005: 195 \\
\hline Ozophora caliginosa Slater & & - & & & & & Baranowski \& Slater, 2005: 196 \\
\hline Ozophora parimpicta Baranowski & & & & & & $\bullet$ & Baranowski \& Slater, 2005: 225 \\
\hline Ozophora quinquemaculata Barber & & $\bullet$ & & & & & Baranowski \& Slater, 2005: 230 \\
\hline \multicolumn{8}{|l|}{ MEMBRACIDAE } \\
\hline Spissistilus festinus (Say) & & - & & & & & Det. Perez-Gelabert (USNM) \\
\hline \multicolumn{8}{|l|}{ MIRIDAE } \\
\hline Eurycipitia clarus (Distant) & & & & - & & & Det. Perez-Gelabert (USNM) \\
\hline \multicolumn{8}{|l|}{ PENTATOMIDAE } \\
\hline Banasa herbacea (Stål) & & & & & $\bullet$ & & $\begin{array}{l}\text { Perez-Gelabert \& Thomas, 2005: } \\
328\end{array}$ \\
\hline Caribo fasciatus Rolston & & & & & $\bullet$ & & $\begin{array}{l}\text { Perez-Gelabert \& Thomas, 2005: } \\
332\end{array}$ \\
\hline Edessa bifida (Say) & $\bullet$ & & & & & & Det. Perez-Gelabert (USNM) \\
\hline Fecelia nigridens (Walker) & $\bullet$ & & & & & & Det. Perez-Gelabert (USNM) \\
\hline Nezara viridula (Linnaeus) & $\bullet$ & & & & & & Det. Perez-Gelabert (USNM) \\
\hline \multicolumn{8}{|l|}{ PYRRHOCORIDAE } \\
\hline Dysdercus sanguinarius Stål & & & & $\bullet$ & & & Det. Perez-Gelabert (USNM) \\
\hline \multicolumn{8}{|l|}{ REDUVIIDAE } \\
\hline Bactrodes sp. & - & & & & & & Det. Perez-Gelabert (USNM) \\
\hline Heza ornata Maldonado Capriles & $\bullet$ & & & & & & $\begin{array}{l}\text { Det. M. C. Coscarön (USNM, } \\
\text { MNHNSD) }\end{array}$ \\
\hline Lophoscutus sp. & & & & $\bullet$ & & & Det. Perez-Gelabert (USNM) \\
\hline Zelus longipes (Linnaeus) & $\bullet$ & & & & & & Det. Perez-Gelabert (USNM) \\
\hline \multicolumn{8}{|l|}{ HYMENOPTERA } \\
\hline \multicolumn{8}{|l|}{ APIDAE } \\
\hline Anthophora hispaniolae Brooks & $\bullet$ & & & & & & Det. J. Genaro (IIBZ) \\
\hline Apis mellifera Linnaeus & $\bullet$ & & & $\bullet$ & & & Det. Perez-Gelabert (USNM) \\
\hline Triepeolus victori Genaro & & & & & & $\bullet$ & Det. J. Genaro (IIBZ) \\
\hline Xylocopa mordax F. Smith & $\bullet$ & & & & & & Det. Perez-Gelabert (IIBZ) \\
\hline \multicolumn{8}{|l|}{ COLLETIDAE } \\
\hline $\begin{array}{l}\text { Caupolicana (Alayoapis) notabilis (F. } \\
\text { Smith) }\end{array}$ & & & & & & $\bullet$ & $\begin{array}{l}\text { Woodruff \& Baranowski (No } \\
\text { publicado) }\end{array}$ \\
\hline \multicolumn{8}{|l|}{ FORMICIDAE } \\
\hline Camponotus christophei W. M. Wheeler & - & & & & & & Det. Perez-Gelabert (IIBZ) \\
\hline Gnamptogenys lineolata Brown & & $\bullet$ & & & & & Brown, 1992: 275 \\
\hline Leptothorax hispaniolae Baroni Urbani & & $\bullet$ & & & & & Baroni Urbani, 1978: 448 \\
\hline Leptothorax sallei (Guérin Méneville) & & $\bullet$ & & & & & Baroni Urbani, 1978: 489 \\
\hline Linepithema keiteli (Forel) & & & & & & & Wild, 2007: 51 \\
\hline \multicolumn{8}{|l|}{ ICHNEUMONIDAE } \\
\hline Enicospilus sp. & - & & & & & & Det. Perez-Gelabert (USNM) \\
\hline \multicolumn{8}{|l|}{ SIRICIDAE } \\
\hline Sirex hispaniola Goulet & & & $\bullet$ & & & & Schiff et al., 2012: 142 \\
\hline \multicolumn{8}{|l|}{ VESPIDAE } \\
\hline $\begin{array}{l}\text { Mischocyttarus mexicanus cubicola Rich- } \\
\text { ards* }\end{array}$ & & & & $\bullet$ & & & Det. S. Navarro (IIBZ) \\
\hline Polistes crinitus (Felton) & $\bullet$ & & & & & & Det. Perez-Gelabert (IIBZ) \\
\hline Polistes minor Palisot de Beauvois & & & & $\bullet$ & & & Det. S. Navarro (IIBZ) \\
\hline \multicolumn{8}{|l|}{ LEPIDOPTERA } \\
\hline \multicolumn{8}{|l|}{ CRAMBIDAE } \\
\hline Agathodes designalis Guenée & & & & & & - & Domínguez, 1997: 71 \\
\hline Azochis rufidiscalis Hampson & & & & & & • & Domínguez, 1997: 71 \\
\hline Cliniodes muralis Hayden & & $\bullet$ & & & & & Hayden, 2011: 262 \\
\hline
\end{tabular}


Tabla II (continuación).

\begin{tabular}{|c|c|c|c|c|c|c|c|}
\hline Especies & $\begin{array}{l}\text { El } \\
\text { Arroyazo }\end{array}$ & \begin{tabular}{|l} 
Loma \\
Casabito
\end{tabular} & La Sal & El Col & \begin{tabular}{|l} 
Loma La \\
Golondrina
\end{tabular} & RCEV & Referencia \\
\hline Desmia funeralis (Hübner) & & & & & & $\bullet$ & Dominguez, 1997: 71 \\
\hline Desmia ploralis (Guenée) & & & & & & $\bullet$ & Domínguez, 1997: 71 \\
\hline Diacme adipaloides (Grote \& Robinson) & & & & & & - & Domínguez, 1997: 71 \\
\hline Diaphania antillia Munroe & & $\bullet$ & $\bullet$ & & & & Domínguez, 1997: 70 \\
\hline Diaphania elegans (Möschler) & & $\bullet$ & & & & & Domínguez, 1997: 70 \\
\hline Diaphania hyalinata (Linnaeus) & & & & & & $\bullet$ & Domínguez, 1997: 71 \\
\hline Diaphania indica (Saunders) & & & & & & - & Domínguez, 1997: 71 \\
\hline Diaphania lualis (Herrich-Schäffer) & & & & & & - & Domínguez, 1997: 71 \\
\hline Epicorsia oedipodalis (Guenée) & & & & & & - & Domínguez, 1997: 71 \\
\hline Glyphodes sibillalis Walker & & & & & & $\bullet$ & Domínguez, 1997: 71 \\
\hline Hellula rogatalis (Hulst) & & & & & & $\bullet$ & Domínguez, 1997: 71 \\
\hline Hymenia perspectalis (Hübner) & & $\bullet$ & $\bullet$ & & & & Domínguez, 1997: 71 \\
\hline Microthyris anormalis (Guenée) & & & & & & $\bullet$ & Domínguez, 1997: 71 \\
\hline Omiodes indicata (Fabricius) & & & & & & • & Domínguez, 1997: 71 \\
\hline Omiodes simialis Guenée & & & & & & $\bullet$ & Domínguez, 1997: 71 \\
\hline Palpita flegia (Cramer) & & & & & & - & Domínguez, 1997: 71 \\
\hline Palpita kimballi Munroe & & & & & & $\bullet$ & Domínguez, 1997: 71 \\
\hline Palpita quadristrigmalis (Guenée) & & & & & & - & Domínguez, 1997: 71 \\
\hline Phaedropsis stictigramma (Hampson) & & & & & & $\bullet$ & Domínguez, 1997: 71 \\
\hline Pyrausta tyralis (Guenée) & & & & & & $\bullet$ & Domínguez, 1997: 71 \\
\hline Samea multiplicalis (Guenée) & & & & & & $\bullet$ & Domínguez, 1997: 71 \\
\hline Spoladea recurvalis (Fabricius) & & & & & & $\bullet$ & Domínguez, 1997: 71 \\
\hline Syngamia florella (Stoll) & & & & & & $\bullet$ & Domínguez, 1997: 71 \\
\hline Terastia meticulosalis Guenée & & & $\bullet$ & & & & Domínguez, 1997: 71 \\
\hline \multicolumn{8}{|l|}{ EREBIDAE } \\
\hline Holomelina semirosea (Druce) & & & & & & $\bullet$ & Domínguez, 1997: 73 \\
\hline Horama panthalon texana (Grote) & & & & & & $\bullet$ & Domínguez, 1997: 73 \\
\hline Lophocampa lineata Vincent & & & & & & $\bullet$ & Vincent, 2011: 71 \\
\hline Lymire edwardsi (Grote) & & & & & & $\bullet$ & Domínguez, 1997: 73 \\
\hline Utetheisa bella (Linnaeus) & & & & & & $\bullet$ & Domínguez, 1997: 72 \\
\hline Westindia haxairei Vincent & & $\bullet$ & & & & $\bullet$ & Vincent et al., 2014: 293 \\
\hline \multicolumn{8}{|l|}{ GEOMETRIDAE } \\
\hline Epimecis hortaria (Fabricius) & & & & & & $\bullet$ & Domínguez, 1997: 72 \\
\hline Epimecis matronaria Guenée & & $\bullet$ & $\bullet$ & & & & Domínguez, 1997: 72 \\
\hline Euchlaena amoenaria astylusaria Walker & & & & & & $\bullet$ & Domínguez, 1997: 72 \\
\hline Nepheloleuca floridata (Grote) & & & & & & - & Domínguez, 1997: 72 \\
\hline Oxydia vesulia transponens (Walker) & & & & & & $\bullet$ & Domínguez, 1997: 72 \\
\hline Renia fraternalis (J. B. Smith) & & - & $\bullet$ & & & & Domínguez, 1997: 72 \\
\hline \multicolumn{8}{|l|}{ HESPERIIDAE } \\
\hline $\begin{array}{l}\text { Eantis papinianus (Poey) } \\
=\text { Achlyodes mihridates }\end{array}$ & & & & & & $\bullet$ & Fernández, 2010: 213 \\
\hline Pyrgus oileus (Linnaeus) & & & & & & $\bullet$ & Domínguez, 1997: 66 \\
\hline \multicolumn{8}{|l|}{ NOCTUIDAE } \\
\hline Agrotis subterranea (Fabricius) & & & & & & $\bullet$ & Domínguez, 1997: 69 \\
\hline Alabama argillacea (Hübner) & & & & & & $\bullet$ & Domínguez, 1997: 69 \\
\hline Anicla infecta (Ochsenheimer) & & & & & & $\bullet$ & Domínguez, 1997: 69 \\
\hline Anomis erosa (Hübner) & & & & & & $\bullet$ & Domínguez, 1997: 69 \\
\hline Anomis flava (Fabricius) & & & & & & $\bullet$ & Domínguez, 1997: 69 \\
\hline Anomis impasta Guenée & & & & & & - & Domínguez, 1997: 69 \\
\hline Anticarsia gemmatalis Hübner & & & & & & $\bullet$ & Domínguez, 1997: 68 \\
\hline Argyrogramma verruca (Fabricius) & & & & & & $\bullet$ & Domínguez, 1997: 70 \\
\hline
\end{tabular}


Tabla II (continuación).

\begin{tabular}{|c|c|c|c|c|c|c|c|}
\hline Especies & \begin{tabular}{l|} 
El \\
Arroyazo
\end{tabular} & $\begin{array}{l}\text { Loma } \\
\text { Casabito }\end{array}$ & La Sal & El Col & $\begin{array}{l}\text { Loma La } \\
\text { Golondrina }\end{array}$ & RCEV & Referencia \\
\hline Ascalapha odorata (Linnaeus) & & & & & & $\bullet$ & Domínguez, 1997: 70 \\
\hline Azeta repugnalis Hübner & & & & & & - & Domínguez, 1997: 68 \\
\hline $\begin{array}{l}\text { Bagisara repanda (Fabricius) } \\
=\text { Atethmia subusta }\end{array}$ & & & & & & - & Domínguez, 1997: 69 \\
\hline Characoma sp. & & & & & & $\bullet$ & Domínguez, 1997: 69 \\
\hline Callopistria floridensis Guenée & & & & & & • & Domínguez, 1997: 69 \\
\hline Condica concisa (Walker) & & & & & & - & Domínguez, 1997: 69 \\
\hline Cydosia nobilitella Cramer & & & & & & $\bullet$ & Domínguez, 1997: 69 \\
\hline Elaphria nucicolora (Guenée) & & & & & & - & Domínguez, 1997: 69 \\
\hline $\begin{array}{l}\text { Feltia jaculifera (Guenée) } \\
=\text { Feltia ducens }\end{array}$ & & & & & & $\bullet$ & Domínguez, 1997: 70 \\
\hline Gonodonta incurva (Sepp) & & & & & & - & Domínguez, 1997: 68 \\
\hline Gonodonta nutrix (Stoll) & & & & & & $\bullet$ & Domínguez, 1997: 68 \\
\hline Gonodonta unica Neumoegen & & & & & & $\bullet$ & Domínguez, 1997: 68 \\
\hline Heliothis subflexa (Guenée) & & & & & & $\bullet$ & Domínguez, 1997: 69 \\
\hline Heliothis virescens (Fabricius) & & & & & & - & Domínguez, 1997: 69 \\
\hline Lesmone formularis (Geyer) & & & & & & • & Domínguez, 1997: 70 \\
\hline Leucania dorsalis Walker & & - & & & & & Adams, 2001: 197 \\
\hline Leucania inconspicua Herrich-Schäffer & & • & & & & & Adams, 2001: 192 \\
\hline Leucania latiuscula Herrich-Schäffer & & - & & & & & Adams, 2001: 200 \\
\hline Leucania lobrega Adams & & - & & & & & Adams, 2001: 189 \\
\hline Leucania rawlinsi Adams & & - & & & & & Adams, 2001: 186 \\
\hline Leucania senescens Möschler & & $\bullet$ & & & & & Adams, 2001: 195 \\
\hline Magusa orbifera (Walker) & & & & & & $\bullet$ & Domínguez, 1997: 69 \\
\hline Melipotis famelica (Guenée) & & & & & & $\bullet$ & Domínguez, 1997: 69 \\
\hline Melipotis fasciolaris (Hübner) & & & & & & $\bullet$ & Domínguez, 1997: 68 \\
\hline Melipotis indomita (Walker) & & & & & & • & Domínguez, 1997: 68 \\
\hline Melipotis januaris (Guenée) & & & & & & $\bullet$ & Domínguez, 1997: 69 \\
\hline Melipotis jucunda Hübner & & & & & & $\bullet$ & Domínguez, 1997: 69 \\
\hline Melipotis perpendicularis (Guenée) & & & & & & - & Domínguez, 1997: 68 \\
\hline Micrathetis triplex (Walker) & & & & & & - & Domínguez, 1997: 69 \\
\hline Mocis disseverans (Walker) & & & & & & - & Domínguez, 1997: 70 \\
\hline Mocis latipes (Guenée) & & & & & & $\bullet$ & Domínguez, 1997: 70 \\
\hline Mythimna unipuncta (Haworth) & & & & & & $\bullet$ & Domínguez, 1997: 69 \\
\hline Panula inconstans (Guenée) & & & & & & $\bullet$ & Domínguez, 1997: 69 \\
\hline Paectes sp. & & & & & & $\bullet$ & Domínguez, 1997: 69 \\
\hline Pseudoplusia includens (Walker) & & & & & & $\bullet$ & Domínguez, 1997: 69 \\
\hline Spodoptera eridania (Cramer) & & & & & & $\bullet$ & Domínguez, 1997: 69 \\
\hline Spodoptera exigua (Hübner) & & & & & & $\bullet$ & Domínguez, 1997: 69 \\
\hline Spodoptera frugiperda (J. E. Smith) & & & & & & - & Domínguez, 1997: 69 \\
\hline Spodoptera latifascia (Walker) & & & & & & $\bullet$ & Domínguez, 1997: 69 \\
\hline Spodoptera ornithogalli (Guenée) & & & & & & - & Domínguez, 1997: 69 \\
\hline Syllectra ericata (Cramer) & & & & & & $\bullet$ & Domínguez, 1997: 69 \\
\hline Thysania zenobia (Cramer) & & & & & & - & Domínguez, 1997: 69 \\
\hline Trichoplusia ni (Hübner) & & & & & & $\bullet$ & Domínguez, 1997: 69 \\
\hline Zale sp. & & & & & & - & Domínguez, 1997: 70 \\
\hline \multicolumn{8}{|l|}{ NOTODONTIDAE } \\
\hline Eremonidia mirifica Rawlins \& Miller & & $\bullet$ & & & & & Rawlins \& Miller, 2008: 207 \\
\hline Nystalea indiana Grote & & & & & & - & Domínguez, 1997: 72 \\
\hline Symmerista albifrons (Smith) & & & & & & - & Domínguez, 1997: 72 \\
\hline \multicolumn{8}{|l|}{ NYMPHALIDAE } \\
\hline Adelpha gelania (Godart) & & & & & & - & Domínguez, 1997: 65 \\
\hline
\end{tabular}


Tabla II (continuación).

\begin{tabular}{|c|c|c|c|c|c|c|c|}
\hline Especies & \begin{tabular}{l|} 
El \\
Arroyazo
\end{tabular} & $\begin{array}{l}\text { Loma } \\
\text { Casabito }\end{array}$ & La Sal & El Col & \begin{tabular}{|l|} 
Loma La \\
Golondrina
\end{tabular} & RCEV & Referencia \\
\hline Agraulis vanillae insularis Maynard & & & & & & $\bullet$ & Domínguez, 1997: 65 \\
\hline Anartia lytrea (Godart) & $\bullet$ & & & & & & Fernánde et al., 2007: 245 \\
\hline Anetia pantheratus (Martyn) & & & & & & $\bullet$ & Domínguez, 1997: 65 \\
\hline $\begin{array}{l}\text { Atlantea cryptadia } \text { Sommer \& Schwartz } \\
=\text { Atlantea } \mathrm{sp} .\end{array}$ & & & & & & - & Domínguez, 1997: 65 \\
\hline Calisto arcas Bates & & & & & & $\bullet$ & Domínguez, 1997: 64 \\
\hline Calisto archebates (Ménétriés) & & & & & & $\bullet$ & Domínguez, 1997: 64 \\
\hline Calisto chrysaoros Bates & & & & & & $\bullet$ & Domínguez, 1997: 64 \\
\hline Calisto pulchella Lathy & & & & & & $\bullet$ & Domínguez, 1997: 64 \\
\hline Dryas iulia fucatus (Boddaert) & & & & & & $\bullet$ & Domínguez, 1997: 65 \\
\hline Greta diaphanus quisqueya (Fox) & $\bullet$ & & & & & $\bullet$ & $\begin{array}{l}\text { Domínguez, 1997: 65; Fernández, } \\
\text { 2010: } 243\end{array}$ \\
\hline $\begin{array}{l}\text { Heliconius charitonia churchi Comstock } \\
\text { \& Brown }\end{array}$ & & & & & & $\bullet$ & Domínguez, 1997: 65 \\
\hline Historis odius (Fabricius) & & & & & & $\bullet$ & Fernández, 2010: 207 \\
\hline Hypanartia paullus (Fabricius) & & & & & & $\bullet$ & Fernández, 2010: 206 \\
\hline Vanessa atalanta rubria (Fruhstorfer) & & & & & & $\bullet$ & Domínguez, 1997: 65 \\
\hline Vanessa virginiensis (Drury) & & & & & & $\bullet$ & Domínguez, 1997: 65 \\
\hline \multicolumn{8}{|l|}{ PIERIDAE } \\
\hline Dysmorphia spio (Godart) & & & & & & $\bullet$ & Domínguez, 1997: 66 \\
\hline Kricogonia lyside (Godart) & & & & & & $\bullet$ & Domínguez, 1997: 66 \\
\hline Zerene cesonia (Butler) & & & & & & $\bullet$ & Domínguez, 1997: 66 \\
\hline \multicolumn{8}{|l|}{ PYRALIDAE } \\
\hline Dioryctria amatella (Hulst) & & & & & & $\bullet$ & Domínguez, 1997: 71 \\
\hline \multicolumn{8}{|l|}{ SPHINGIDAE } \\
\hline Agrius cingulatus (Fabricius) & & & & & & $\bullet$ & Domínguez, 1997: 67 \\
\hline Amphoxyx kofleri Eitschberger & & $\bullet$ & & & & & Eitschberger, 2006: 177 \\
\hline Cocytius antaeus antaeus (Drury) & & & & & & $\bullet$ & Domínguez, 1997: 67 \\
\hline Cocytius duponchel (Poey) & & & & & & $\bullet$ & Domínguez, 1997: 67 \\
\hline Enyo lugubris lugubris (Linnaeus) & & & & & & $\bullet$ & Domínguez, 1997: 68 \\
\hline Erinnyis alope alope (Drury) & & & & & & $\bullet$ & Domínguez, 1997: 67 \\
\hline Erinnyis crameri (Schaus) & & & & & & $\bullet$ & Domínguez, 1997: 67 \\
\hline Erinnyis ello ello (Linnaeus) & & & & & & $\bullet$ & Domínguez, 1997: 67 \\
\hline Erinnyis guttularis (Walker) & & & & & & $\bullet$ & Domínguez, 1997: 67 \\
\hline Erinnyis lassauxii (Boisduval) & & & & & & $\bullet$ & Domínguez, 1997: 67 \\
\hline Eumorpha fasciata fasciata (Sulzer) & & & & & & $\bullet$ & Domínguez, 1997: 68 \\
\hline Eumorpha labruscae labruscae (Linnaeus) & & & & & & $\bullet$ & Domínguez, 1997: 68 \\
\hline Eumorpha vitis vitis (Linnaeus) & & & & & & $\bullet$ & Domínguez, 1997: 68 \\
\hline Hyles lineata lineata (Fabricius) & & & & & & $\bullet$ & Domínguez, 1997: 68 \\
\hline $\begin{array}{l}\text { Isognathus rimosus molitor Rothschild \& } \\
\text { Jordan }\end{array}$ & & & & & & $\bullet$ & Domínguez, 1997: 67 \\
\hline Manduca quinquemaculata (Haworth) & & & & & & $\bullet$ & Domínguez, 1997: 67 \\
\hline Manduca rustica dominicana (Gehlen) & & & & & & $\bullet$ & Domínguez, 1997: 67 \\
\hline Manduca sexta jamaicensis (Linnaeus) & & & & & & $\bullet$ & Domínguez, 1997: 67 \\
\hline Neococytius cluentius (Cramer) & & & & & & $\bullet$ & Eitschberger, 2006: 197 \\
\hline Pachylia ficus (Linnaeus) & & & & & & $\bullet$ & Domínguez, 1997: 67 \\
\hline Pachylioides resumens (Walker) & & & & & & $\bullet$ & Domínguez, 1997: 67 \\
\hline Perigonia lusca lusca (Fabricius) & & & & & & $\bullet$ & Domínguez, 1997: 68 \\
\hline Protambulyx strigilis strigilis (Linnaeus) & & & & & & $\bullet$ & Domínguez, 1997: 67 \\
\hline Pseudosphinx tetrio (Linnaeus) & & & & & & $\bullet$ & Domínguez, 1997: 67 \\
\hline Xylophanes chiron necchus (Cramer) & & & & & & $\bullet$ & Domínguez, 1997: 68 \\
\hline Xylophanes falco (Walker) & & & & & & $\bullet$ & Domínguez, 1997: 68 \\
\hline Xylophanes tersa tersa (Linnaeus) & & & & & & $\bullet$ & Domínguez, 1997: 68 \\
\hline
\end{tabular}


Tabla II (continuación).

\begin{tabular}{|c|c|c|c|c|c|c|c|}
\hline Especies & $\begin{array}{l}\text { El } \\
\text { Arroyazo }\end{array}$ & $\begin{array}{l}\text { Loma } \\
\text { Casabito }\end{array}$ & La Sal & $\mathrm{El} \mathrm{Col}$ & $\begin{array}{l}\text { Loma La } \\
\text { Golondrina }\end{array}$ & RCEV & Referencia \\
\hline \multicolumn{8}{|l|}{ MANTODEA } \\
\hline \multicolumn{8}{|l|}{ MANTIDAE } \\
\hline Callimantis antillarum (Saussure) & $\bullet$ & & & & & & $\begin{array}{l}\text { Lombardo \& Perez-Gelabert, } \\
\text { 2004: } 40\end{array}$ \\
\hline Gonatista sp. & - & & & & & & Det. D. Perez-Gelabert (USNM) \\
\hline \multicolumn{8}{|l|}{ ODONATA } \\
\hline \multicolumn{8}{|l|}{ COENAGRIONIDAE } \\
\hline Enallagma civile (Hagen) & $\bullet$ & & & & & & Det. A. Sánchez (IIBZ) \\
\hline Enallagma coecum (Hagen) & $\bullet$ & & $\bullet$ & & & & Det. A. Sánchez (IIBZ) \\
\hline Telebasis dominicana (Selys) & $\bullet$ & $\bullet$ & & & & & Det. A. Sánchez (IIBZ) \\
\hline Telebasis vulnerata (Hagen) & $\bullet$ & & $\bullet$ & & & & Det. A. Sánchez (IIBZ) \\
\hline \multicolumn{8}{|l|}{ MEGAPODAGRIONIDAE } \\
\hline $\begin{array}{l}\text { Hypolestes hatuey Torres-Cambas } \\
=\text { Hypolestes clara (Calvert) error id. }\end{array}$ & - & & & & & $\bullet$ & $\begin{array}{l}\text { Fernández, 2007: 236; Fernández, } \\
\text { 2010: } 124\end{array}$ \\
\hline \multicolumn{8}{|l|}{ PROTONEURIDAE } \\
\hline Protoneura dunklei Daigle & & & $\bullet$ & & & & Det. A. Sánchez (IIBZ) \\
\hline Protoneura sanguinipes Westfall & $\bullet$ & & & & & & Det. A. Sánchez (IIBZ) \\
\hline \multicolumn{8}{|l|}{ SYNLESTIDAE } \\
\hline Phylolestes ethelae Christiansen & & & $\bullet$ & & & & Det. A. Sánchez (IIBZ) \\
\hline \multicolumn{8}{|l|}{ AESHNIDAE } \\
\hline Gynacantha nervosa Rambur & - & & & & & & Det. A. Sánchez (IIBZ) \\
\hline Rhionaeschna psilus (Calvert) & & & $\bullet$ & & & & Det. A. Sánchez (IIBZ) \\
\hline \multicolumn{8}{|l|}{ GOMPHIDAE } \\
\hline Macrothemis celeno (Selys) & $\bullet$ & & & & & & Det. A. Sánchez (IIBZ) \\
\hline Progomphus serenus Hagen & - & & $\bullet$ & & & & Det. A. Sánchez (IIBZ) \\
\hline Progomphus zephyrus Needham & & & & & & & Det. A. Sánchez (IIBZ) \\
\hline \multicolumn{8}{|l|}{ LIBELLULIDAE } \\
\hline Erythrodiplax umbrata (Linnaeus) & $\bullet$ & & & & & & Det. A. Sánchez (IIBZ) \\
\hline Pantala flavescens Fabricius & & & & $\bullet$ & & & Det. A. Sánchez (IIBZ) \\
\hline Scapanea frontalis (Burmeister) & $\bullet$ & & $\bullet$ & & & & Det. A. Sánchez (IIBZ) \\
\hline Sympetrum gilvum (Hagen) & $\bullet$ & & - & & & & Det. A. Sánchez (IIBZ) \\
\hline \multicolumn{8}{|l|}{ ORTHOPTERA } \\
\hline \multicolumn{8}{|l|}{ ACRIDIDAE } \\
\hline $\begin{array}{l}\text { Dellia roseomaculata } \text { Perez-Gelabert \& } \\
\text { Otte }\end{array}$ & & & & $\bullet$ & & & Det. D. Perez-Gelabert (USNM) \\
\hline Hispanacris oreades Perez et al. & & & $\bullet$ & & & & Perez-Gelabert et al., 1995: 165 \\
\hline Orphulella nesicos Otte & & & $\bullet$ & & & & Det. D. Perez-Gelabert (USNM) \\
\hline Orphulella punctata (De Geer) & $\bullet$ & & & & & & Det. D. Perez-Gelabert (USNM) \\
\hline Schistocerca quisqueya Rehn \& Hebard & $\bullet$ & & & & & & Det. D. Perez-Gelabert (USNM) \\
\hline $\begin{array}{l}\text { Tergoceracris ebanoverde Perez-Gelabert } \\
\& \text { Otte }\end{array}$ & $\bullet$ & $\bullet$ & $\bullet$ & & & & Perez-Gelabert \& Otte, 2003: 5 \\
\hline \multicolumn{8}{|l|}{ EPISACTIDAE } \\
\hline Antillacris explicatrix Rehn \& Rehn & - & $\bullet$ & & & • & & $\begin{array}{l}\text { Perez-Gelabert \& Rowell, 2006: } \\
243\end{array}$ \\
\hline Espagnola darlingtoni Rehn \& Rehn & & & $\bullet$ & $\bullet$ & & & $\begin{array}{l}\text { Perez-Gelabert \& Rowell, 2006: } \\
245\end{array}$ \\
\hline Espagnolopsis breviptera Perez et al. & $\bullet$ & & $\bullet$ & & $\bullet$ & & Perez-Gelabert et al., 1997: 159 \\
\hline \multicolumn{8}{|l|}{ GRYLLIDAE } \\
\hline Amphiacusta annulipes (Serville) & $\bullet$ & & $\bullet$ & & & & Otte \& Perez-Gelabert, 2009: 588 \\
\hline $\begin{array}{l}\text { Amphiacusta dissimilis Otte \& Perez-Ge- } \\
\text { labert }\end{array}$ & & & $\bullet$ & & & & Otte \& Perez-Gelabert, 2009: 584 \\
\hline Antillicharis domingensis (Saussure) & $\bullet$ & & $\bullet$ & & & & Otte \& Perez-Gelabert, 2009: 246 \\
\hline Antillicharis vigil Otte \& Perez-Gelabert & $\bullet$ & & & & & & Otte \& Perez-Gelabert, 2009: 247 \\
\hline Chremon procax Otte \& Perez-Gelabert & $\bullet$ & & - & & & & Otte \& Perez-Gelabert, 2009: 487 \\
\hline
\end{tabular}


Tabla II (continuación).

\begin{tabular}{|c|c|c|c|c|c|c|c|}
\hline Especies & $\begin{array}{l}\text { El } \\
\text { Arroyazo }\end{array}$ & $\begin{array}{l}\text { Loma } \\
\text { Casabito }\end{array}$ & La Sal & El Col & \begin{tabular}{l|} 
Loma La \\
Golondrina
\end{tabular} & RCEV & Referencia \\
\hline Cyrtoxypha eucrines Otte \& Perez-Gelabert & $\bullet$ & & $\bullet$ & & & & Otte \& Perez-Gelabert, 2009: 145 \\
\hline Gryllus assimilis Fabricius & $\bullet$ & $\bullet$ & $\bullet$ & & & & Otte \& Perez-Gelabert, 2009: 37 \\
\hline Hapithus cerbatana Otte \& Perez-Gelabert & & & • & & & & Otte \& Perez-Gelabert, 2009: 228 \\
\hline Hapithus mythicos Otte \& Perez-Gelabert & $\bullet$ & & & & & & Otte \& Perez-Gelabert, 2009: 228 \\
\hline Hygronemobius epia Otte \& Perez-Gelabert & $\bullet$ & & $\bullet$ & & & & Otte \& Perez-Gelabert, 2009: 105 \\
\hline Knyella vivax Otte \& Perez-Gelabert & $\bullet$ & & - & & & & Otte \& Perez-Gelabert, 2009: 237 \\
\hline Margarettia guajey Otte \& Perez-Gelabert & $\bullet$ & & $\bullet$ & & & & Otte \& Perez-Gelabert, 2009: 235 \\
\hline $\begin{array}{l}\text { Pteronemobius setiger Otte \& Perez- } \\
\text { Gelabert }\end{array}$ & - & & & & & & Otte \& Perez-Gelabert, 2009: 108 \\
\hline Stenogryllus phthisicus Saussure & & & $\bullet$ & & & & Otte \& Perez-Gelabert, 2009: 477 \\
\hline Tafalisca lurida Walker & & & $\bullet$ & & & & Otte \& Perez-Gelabert, 2009: 482 \\
\hline Walkerella encollos Otte \& Perez-Gelabert & $\bullet$ & & & & & & Otte \& Perez-Gelabert, 2009: 258 \\
\hline Walkerella ochleros Otte \& Perez-Gelabert & & & $\bullet$ & & & & Otte \& Perez-Gelabert, 2009: 258 \\
\hline Walkerella timens Otte \& Perez-Gelabert & $\bullet$ & & & & & & Otte \& Perez-Gelabert, 2009: 258 \\
\hline Yoyuteris barahona Ruíz \& Otte & & & $\bullet$ & & & & Otte \& Perez-Gelabert, 2009: 675 \\
\hline Yoyuteris cerritus Otte \& Perez-Gelabert & & & $\bullet$ & & & & Otte \& Perez-Gelabert, 2009: 674 \\
\hline \multicolumn{8}{|l|}{ TETTIGONIIDAE } \\
\hline Anaulocomera sp. & $\bullet$ & & & & & & Perez-Gelabert, 2001: 68 \\
\hline Conocephalus cinereus Thunberg & $\bullet$ & & & & & & Det. D. Perez-Gelabert (USNM) \\
\hline Erechthis gundlachi Bolívar & $\bullet$ & & $\bullet$ & & & & Perez-Gelabert, 2014: 38 \\
\hline Neoconocephalus sp. & $\bullet$ & & $\bullet$ & & & & Det. D. Perez-Gelabert (USNM) \\
\hline $\begin{array}{l}\text { Polyancistrus serrulatus (Palisot de Beau- } \\
\text { vois) }\end{array}$ & $\bullet$ & & & & & & Det. D. Perez-Gelabert (USNM) \\
\hline Spelaeala sp. & - & & $\bullet$ & & & & Det. D. Perez-Gelabert (USNM) \\
\hline Turpilia sp. 1 & $\bullet$ & & & & & & Det. D. Perez-Gelabert (USNM) \\
\hline Turpilia sp. 2 & $\bullet$ & & & & & & Det. D. Perez-Gelabert (USNM) \\
\hline \multicolumn{8}{|l|}{ PHASMATODEA } \\
\hline \multicolumn{8}{|l|}{ PHASMATIDAE } \\
\hline Taraxippus paliurus Moxey & & & $\bullet$ & & & & Fernández, 2007: 247 \\
\hline \multicolumn{8}{|l|}{ TRICHOPTERA } \\
\hline \multicolumn{8}{|l|}{ CALAMOCERATIDAE } \\
\hline Phylloicus iridescens Banks & & - & & & & & Flint \& Sykora, 2004: 3 \\
\hline \multicolumn{8}{|l|}{ HELICOPSYCHIDAE } \\
\hline $\begin{array}{l}\text { Helicopsyche dominicana Botosaneanu } \\
\& \text { Flint }\end{array}$ & $\bullet$ & & & & & & Det. O. S. Flint (USNM) \\
\hline Helicopsyche parahageni Flint \& Sykora & $\bullet$ & & & & & & Det. O. S. Flint (USNM) \\
\hline \multicolumn{8}{|l|}{ HYDROBIOSIDAE } \\
\hline Atopsyche peravia Flint \& Sykora & & $\bullet$ & & & & & Flint \& Sykora, 2004: 16 \\
\hline \multicolumn{8}{|l|}{ HYDROPSYCHIDAE } \\
\hline Calosopsyche carinifera (Flint) & & $\bullet$ & & & & & Flint \& Sykora, 2004: 18 \\
\hline Calosopsyche domingensis (Banks) & $\bullet$ & - & & & & & Flint \& Sykora, 2004: 19 \\
\hline \multicolumn{8}{|l|}{ GLOSSOSOMATIDAE } \\
\hline Cariboptila hispaniolica Flint & - & & & & & & Flint \& Sykora, 2004: 6 \\
\hline \multicolumn{8}{|l|}{ LEPTOCERIDAE } \\
\hline Nectopsyche cubana (Banks) & $\bullet$ & & & & & & Det. O. S. Flint (USNM) \\
\hline \multicolumn{8}{|l|}{ PHILOPOTAMIDAE } \\
\hline Chimarra (Chimarrita) merengue Blahnik & & $\bullet$ & & & & & Flint \& Sykora, 2004: 51 \\
\hline Chimarra (Curgia) gilvimacula Flint & $\bullet$ & & & & & & Det. O. S. Flint (USNM) \\
\hline \multicolumn{8}{|l|}{ POLYCENTROPODIDAE } \\
\hline Polycentropus jeldesi Flint & & $\bullet$ & & & & & Flint \& Sykora, 2004: 54 \\
\hline Polycentropus marcanoi Flint & $\bullet$ & & & & & & Det. O. S. Flint (USNM) \\
\hline Polycentropus vanderpooli Flint & $\bullet$ & & & & & & Det. O. S. Flint (USNM) \\
\hline
\end{tabular}




\section{RECOMENDACIONES}

Se recomienda la realización de un inventario entomológico básico concentrado en la RCEV, orientado a grupos específicos para los cuales se disponga de taxónomos expertos. Tal inventario aumentaría de manera considerable los reportes de especies que habitan en esta reserva y permitiría tener una mejor visión de conjunto de sus ecosistemas.

\section{AGRADECIMIENTOS}

A Ramón Elías Castillo (Fundación PROGRESSIO) por su constante apoyo a nuestros trabajos de campo en la RCEV y comentarios sobre este manuscrito. También se agradece la cordial y oportuna ayuda de Ramona Ortíz y los guardaparques de la reserva en las estaciones de El Arroyazo, La Sal, Loma Casabito y El Col. Los datos sobre Odonata fueron contribuidos por América Sánchez y Ruth Bastardo (IIBZ). Gavino Guzmán (Centro Nacional de Enfermedades Tropicales, Santo Domingo) identificó las dos especies de mosquitos. Santo Navarro (IIBZ) y Julio A. Genaro (Toronto) ayudaron con algunas determinaciones de himenópteros y Oliver Flint, Jr. (USNM) aportó varios nuevos registros de tricópteros. Sardis Medrano (Instituto Dominicano de Investigaciones Agrícolas y Forestales, Santo Domingo) contribuyó las determinaciones de varias especies de Phyllophaga. También se agradece la compañía y ayuda con las colectas de Brígido Hierro, Ruth Bastardo, América Sánchez, Carlos de Soto Molinari y Juan Sánchez Borbón. Una versión preliminar de este manuscrito fue mejorada por correcciones y sugerencias de Sheyla Yong (Universidad de La Habana, Cuba) y Ruth Bastardo (IIBZ).

\section{LITERATURA CITADA}

Baranowski, R. M. y J. A. Slater. 2005. The Lygaeidae of the West Indies. University of Florida, IFAS, Florida Agricultural Experiment Station, Bulletin, 402, 266 pp.

Baroni Urbani, C. 1978. Materiali per una revision dei Leptothorax neotropicali appartenenti al sottogenere Macromischa Roger, n. comb. (Hymenoptera: Formicidae). Entomologica Basiliensia, 3: 395-618.

Bell, R. T. y J. R. Bell. 1985. Rhysodini of the world part IV. Revisions of Rhyzodiastes Fairmaire and Clinidium Kirby, with new species in other genera (Coleoptera: Carabidae or Rhysodidae). Quaestiones Entomologicae, 21: 1-172.

Brown, W. L., Jr. 1992. Two new species of Gnamptogenys, and an account of millipede predation by one of them. Psyche, 99: 275-289.

Campbell, J. M. 1978. New species and records of West Indian Alleculidae (Coleoptera). Studies on Neotropical Fauna and Environment, 13: 203-212.

Castillo Torre, R. E., T. May y H. L. Domínguez. 1997. Estudios sobre fauna y flora de la Reserva Científica Ébano Verde. Santo Domingo, República Dominicana. Serie Publicaciones PROGRESSIO, 14.

Chaboo, C. S. 2000. Revision and phylogeny of the Caribbean genus Elytrogona Chevrolat (Coleoptera: Chrysomelidae: Cassidinae: Stolaini). The Coleopterists' Bulletin, 54: 379394.

Chapman, A. D. 2009. Numbers of Living Species in Australia and the World. $2^{\text {nd }}$ Edition. Report for the Australian Biological Resources Study, Canberra, Australia, 78 pp. 
Domínguez, H. L. 1997. Las mariposas de la Reserva Científica Ébano Verde. Estudios Sobre Fauna y Flora de la Reserva Científica Ébano Verde, Serie Progressio, 14: 63-74.

Eitschberger, U. 2006. Revision der Gattungen Amphimoea Rothschild \& Jordan, 1903, Cocytius auct. (nec Hubner, [1819]) und Neococytius Hodges, 1971 mit der Neugliederung der Gattung Cocytius auct. Neue Entomologische Nachrichten, 59: 171-288.

Fernández, E. 2010. Reserva Científica Ébano Verde, un Encuentro con la Naturaleza. Editora Amigo del Hogar, Santo Domingo, 227 pp.

Fernández, E., E. O. Wilson, P. Bayard, B. Farrell, S. Latta, C. Rimmer, T. Baroni, C. Woods, J. A. Ottenwalder, S. Cantrell, R. García, M. Mejía y B. Hedges. 2007. Hispaniola, A Photographic Journey Through Island Biodiversity. Harvard University Press, Cambridge, 396 pp.

Flint, O. S., Jr. y J. Sykora, J. 2004. Caddisflies of Hispaniola, with special reference to the Dominican Republic (Insecta: Trichoptera). Annals of the Carnegie Museum, 73: 1-60.

Franz, H. 1984. Scydmaeniden von den Karibischen Inseln (Dominikanische Republik, Puerto Rico, San Tomas und Gouadeloupe) (Scydmaenidae, Col.). Koleopterologische Rundschau, 57: $15-34$.

Freytag, P. H. 2005a. The Gyponinae of Hispaniola (Hemiptera: Cicadellidae). Journal of the Kansas Entomological Society, 78: 322-340.

Freytag, P. H. 2005b. Review of the genus Caribovia Young (Hemiptera: Cicadellidae), including thirteen new species. Journal of the Kansas Entomological Society, 78: 341-362.

Freytag, P. H. 2007. Two new genera and six new species of leafhoppers (Hemiptera: Cicadellidae: Cicadellinae) from Hispaniola. Entomological News, 118: 385-396.

Freytag, P. H. 2009. Review of the genus Agalliopsis for Hispaniola (Hemiptera: Cicadellidae: Agalliinae), including thirteen new species. Entomological News, 120: 260-278.

Freytag, P. H. 2012. A review of the New World Krisnini (Hemiptera: Cicadellidae: Iassinae) including three genera and six new species. Insecta Mundi, 0246: 1-8.

García, R., M. Mejía y T. Zanoni. 1994. Composición florística y principales asociaciones vegetales de la Reserva Científica Ébano Verde, Cordillera Central, República Dominicana. Moscosoa, 8: 86-130.

Gutiérrez, E. 2013. El género Eurycotis (Dictyoptera: Blattaria: Blattidae: Polyzosterinae) I. Especies de la Hispaniola. Primera parte: seis especies nuevas. Novitates Caribaea, 6: 16-35.

Gutiérrez, E. y D. E. Perez-Gelabert. 2000. Annotated checklist of Hispaniolan cockroaches. Transactions of the American Entomological Society, 126: 423-445.

Gutiérrez-Fonseca, P. E., K. G. Rosas y A. Ramírez. 2013. Aquatic insects of Puerto Rico: a list of families. Dugesiana, 20: 215-219.

Hayden, J. E. 2011. Revision of Cliniodes Guenée (Lepidoptera: Crambidae: Odontiinae). Annals of the Carnegie Museum, 79: 231-347. 
Hilario Pérez, A. D. 2013. Evaluación rápida de los invertebrados del Parque Nacional Aniana Vargas, Cotuí, Provincia Sánchez Ramírez. Anuario de Divulgación Científica 2012, UASD, $1(1): 8-16$.

Hopp, K. J. y M. A. Ivie. 2009. A revision of the West Indian genus Nesocyrtosoma Marcuzzi (Coleoptera: Tenebrionidae). The Coleopterists' Society Monographs, Supplement to The Coleopterists' Bulletin, 63: 1-138.

Howden, H. F. 1978. Descriptions of some West Indian Scarabaeidae primarily in the Natural History Museum, Basel. Entomologica Basiliensia, 3: 377-393.

Kazantsev, S. V. y D. E. Perez-Gelabert. 2009. Fireflies of Hispaniola (Coleoptera: Lampyridae). Russian Entomological Journal, 17: 367-402.

Lingafelter, S. W. 2010. Methiini and Oemini (Coleoptera: Cerambycidae: Cerambycinae) of Hispaniola. The Coleopterists' Bulletin, 64: 265-269.

Lingafelter, S. W. 2011. New records of Caribbomerus from Hispaniola and Dominica with redescription of $\mathrm{C}$. elongatus (Fisher) and a key to species of the genus in the West Indies (Coleoptera, Cerambycidae, Cerambycinae, Graciliini). ZooKeys, 85: 27-39.

Lingafelter, S. W. y C. Micheli. 2009. The genus Leptostylopsis of Hispaniola (Coleoptera, Cerambycidae, Acanthocinini). ZooKeys, 17: 1-55.

Lombardo, F. y D. E. Perez-Gelabert. 2004. The mantids of Hispaniola, with the description of two new species (Mantodea). Boletín de la Sociedad Entomológica Aragonesa, 34: 35-48.

Mateo Jiménez, A. L. 2011. Diversidad de musgos (Bryophyta) de la Reserva Científica Ébano Verde, La Vega, República Dominicana. Tesis de Licenciatura en Biología, Universidad Autónoma de Santo Domingo, República Dominicana. 73 pp.

Medvedev, L. N. 2004. Notes on Neotropical Alticinae (Coleoptera, Chrysomelidae). Bonner Zoologische Beiträge, 52: 121-125.

Mengual, X. 2014. Revision of the genus Rhinoprosopa. The Canadian Entomologist, 147: 1-22.

Navarro, L., T. M. Aide, M. G. Slocum y J. K. Zimmerman. 2006. Restauración de Especies Nativas en los Helechales de la Reserva Ébano Verde. Editora Amigo del Hogar, Santo Domingo, $137 \mathrm{pp}$.

Opitz, W. 2008. Classification, natural history, and evolution of Epiphloeinae (Coleoptera: Cleridae). Part V. Decorosa Opitz, a new genus of checkered beetles from Hispaniola with description of its four new species. American Museum Novitates, 3628: 1-19.

Otte, D. y D. E. Perez-Gelabert. 2009. Caribbean Crickets. Publications on Orthoptera Diversity, The Orthopterists' Society, Philadelphia, 792 pp.

Perez-Gelabert, D. E. 2001. Preliminary checklist of the Orthoptera (Saltatoria) of Hispaniola. Journal of Orthoptera Research, 10: 63-74.

Perez-Gelabert, D. E. 2008. Arthropods of Hispaniola (Dominican Republic and Haiti): A checklist and bibliography. Zootaxa, 1831: 1-530. 
Perez-Gelabert, D. E. 2014. Two new records of katydids (Orthoptera: Tettigoniidae: Conocephalinae) from the Dominican Republic, Hispaniola. Novitates Caribaea, 7: 37-43.

Perez-Gelabert, D. E., G. O. Dominici, B. Hierro y D. Otte. 1995. New grasshopper genera and species from the Dominican Republic (Hispaniola) (Acridoidea: Acrididae). Transactions of the American Entomological Society, 121: 153-171.

Perez-Gelabert, D. E., B. Hierro y D. Otte. 1997. Espagnolopsis and Espagnolina, two new genera of eumastacid grasshoppers (Orthoptera: Eumastacidae: Episactinae) from Hispaniola. Journal of Orthoptera Research, 6: 153-160.

Perez-Gelabert, D. E. y D. Otte. 2003. Tergoceracris, a new genus and six new species of montane grasshoppers (Orthoptera: Acrididae: Ommatolampinae) from Dominican Republic and Puerto Rico. Zootaxa, 155: 1-31.

Perez-Gelabert, D. E. y C. H. F. Rowell. 2006. Further investigations of Hispaniolan eumastacoid grasshoppers (Espagnolinae: Episactidae: Orthoptera). Journal of Orthoptera Research, 15: 241-249.

Perez-Gelabert, D. E. y D. B. Thomas. 2005. Stink bugs (Heteroptera: Pentatomidae) of the island of Hispaniola, with seven new species from the Dominican Republic. Boletín de la Sociedad Entomológica Aragonesa, 37: 319-352.

Perez-Gelabert, D. E, R. H. Bastardo y S. Medrano. 2011. Entomofauna del Parque Nacional Loma Nalga de Maco y alrededores, provincia Elías Piña, República Dominicana. Novitates Caribaea, 4: 80-90.

Prena, J. 2009. The West Indian Peridinetini (Coleoptera: Curculionidae: Baridinae). Zootaxa, 2210: 51-64.

Quírico Batista, M. E. 2004. Basidiomycetes de la Reserva Científica Ébano Verde (Cordillera Central), República Dominicana. Tesis de Licenciatura en Biología, Universidad Autónoma de Santo Domingo, República Dominicana. 114 pp + Anexos.

Rawlins, J. E. y J. S. Miller. 2008. Dioptine moths of the Caribbean region: description of two new genera with notes on biology and biogeography (Lepidoptera: Notodontidae: Dioptinae). Annals of the Carnegie Museum, 76: 203-225.

Rodríguez, K., F. Paz y R. H. Bastardo. 2014. Diversidad y patrones de distribución de las mariposas diurnas (Lepidoptera: Papilionoidea y Hesperioidea) en un transecto altitudinal del Parque Nacional Sierra Martín García, República Dominicana. Novitates Caribaea, 7: 72-82.

Scarbrough, A. G. y D. E. Perez-Gelabert. 2003. Ommatius Wiedemann (Diptera: Asilidae) from Hispaniola: five new species and a modified key, phenology, and distribution records of species. Boletín de la Sociedad Entomológica Aragonesa, 33: 41-58.

Scarbrough, A. G., D. E. Perez-Gelabert y S. H. Page. 2005. Synopsis of leptogastrine asilids (Diptera) from Hispaniola. Transactions of the American Entomological Society, 131: 29-67. 
Schiff, N.M., H. Goulet, D. R. Smith, C. Boudreault, A. D. Wilson y B. E. Scheffler, 2012. Siricidae (Hymenoptera: Symphyta: Siricoidea) of the Western Hemisphere. Canadian Journal of Arthropod Identification, 21: 1-305.

Skelley, P. E. 2009. Pleasing fungus beetles of the West Indies (Coleoptera: Erotylidae: Erotylinae). Insecta Mundi, 0082: 1-94.

Soldner, M., I. Stephen, L. Ramos, R. Angus, N. C. Wells, A. Grosso y M. Crane. 2004. Relationship between macroinvertebrate fauna and environmental variables in small streams of the Dominican Republic. Water Research, 38: 863-874.

Stebnicka, Z. 2004. The New World species of Ataenius Harold, 1867. IV. Revision of the A. strigicauda group (Coleoptera: Scarabaeidae: Aphodiinae: Eupariini). Acta Zoologica Cracoviensia, 47: 211-228.

Triplehorn, C.A. y O. Merkl. 1997. Review of the genus Loxostethus Triplehorn, with descriptions of three new species (Coleoptera: Tenebrionidae: Diaperini). Annals of the Entomological Society of America, 90: 736-741.

Vincent, B. 2011. Description of two new Lophocampa Harris from the Dominican Republic (Arctiidae, Arctiinae). ZooKeys, 75: 69-77.

Vincent, B., M. Hajibabaei y R. Rougerie. 2014. A striking new genus and species of tigermoth (Lepidoptera: Erebidae, Arctiinae, Arctiini) from the Caribbean, with molecular and morphological analysis of its systematic placement. Zootaxa, 3760 (2): 289-300.

Wild, A. L. 2007. Taxonomic revision of the ant genus Linepithema (Hymenoptera: Formicidae). University of California Publications in Entomology, 126: 1-151.

Wittmer, W. 1992. Zur Kenntnis der Gattung Tytthonyx Le Conte, 1851. Beitrag 2. (Coleoptera: Cantharidae, Subfam. Silinae, tribus Tytthonyxini). Entomologica Basiliensia, 15: 333-377.

Woodruff, R. E. y R. M. Baranowski. 2000. Report for Dirección Nacional de Parques de la República Dominicana. [unpublished].

Woodruff, R. E. y M. W. Sanderson. 2004. Revision of the Phyllophaga of Hispaniola (Coleoptera: Scarabaeidae: Melolonthinae). Insecta Mundi, 18: 1-154.

[Recibido: 12 de agosto, 2015. Aceptado para publicación: 30 de septiembre, 2015] 